\title{
MITOCHONDRIAL DYSFUNCTION IN EARLY DIAGNOSED AUTISM SPECTRUM DISORDER CHILDREN
}

\author{
SHAHJADI $\mathrm{S}^{1}$, KHAN AS ${ }^{2}$, AHMED MU 3
}

\begin{abstract}
:
Background: Mitochondrial dysfunction and abnormal brain bioenergetics can cause autism. Cellular function impairment due to mitochondrial dysfunction may cause cognitive impairment, language deficits and abnormal energy metabolism in autism. Objective: The aim of this study was to evaluate biochemical evidence of the mitochondrial dysfunction by measuring blood ammonia, serum lactate, alanine aminotransferase (ALT), aspartate aminotransferase (AST) and creatinine kinase (CK) in autism spectrum disorder children.
\end{abstract}

Methods: This observational type of analytical study with case-control design was conducted in the Department of Physiology of Bangabandhu Sheikh Mujib Medical University (BSMMU), Shahbag, Dhaka. For this study, a total number of 20 Subjects were randomly selected, among which 10 were apparently healthy subjects (control group-A) for comparison and 10 were diagnosed children with autism spectrum disorder (study group- $B$ ). $5 \mathrm{ml}$ venous blood was collected from both groups for analysis serum CK, AST, ALT, lactate and blood ammonia. Blood ammonia, serum lactate, AST, ALT and CK level were estimated in all children by standard laboratory method. Independent sample ' $t$ ' test was used for statistical analysis. $P$ value $<0.05$ was accepted as significant. The mean of all the measured biochemical variables in normal children were within normal ranges.

Result: Blood ammonia, serum lactate, AST, CK were found significantly higher in autism spectrum disorder children in comparison to control.

Conclusion: From the result of this study it may be concluded that mitochondrial dysfunction occur in autistic spectrum disorder children .

Key words: autism spectrum disorder, mitochondrial dysfunction, blood ammonia, serum lactate.

J Dhaka Med Coll. 2017; 26(1) : 43-47

\section{Introduction}

Impaired social interaction, communication and imaginative skills as well as stereotyped, bizarre behaviour patterns and interests characterize autistic syndromes. The children have a restricted number of interests $1,2,3$. According to the American Psychiatric Association (1994), autism is associated with behavioral, developmental, neuropathological and sensory abnormalities. Autism is usually diagnosed between mthe ages of 2 to 10 years of age, with peak prevalence between 5 to 8 years of age ${ }^{4}$. A group of common developmental disorders are called Autism Spectrum Disorders (ASD). The word spectrum refers to a wide range of disorders having different domains according to the severity of features ${ }^{5}$. Autistic disorders have three core symptomatic domains: deficits in communication, abnormal social interaction, and restrictive or repetitive interests and behaviors ${ }^{6}$. Autism is a metabolic disorder that ultimately affects the CNS and its function ${ }^{7}$. Mitochondrial dysfunction is one of the important causes of autism spectrum disorder. A recent study revealed that $80 \%$ of children with autism spectrum disorder may

1. Dr. Shorifa Shahjadi, Assistant Professor, Department of Physiology, Bangabandhu Sheikh Mujib Medical University, Dhaka.

2. Dr. Arif Salam Khan, Associate Professor, Department of Surgery, Bangabandhu Sheikh Mujib Medical University, Dhaka.

3. Dr. Mesbah Uddin Ahmed, Professor and Head of the Department, Department of Paediatrics, Samaj Vittik Medical college, Savar, Dhaka

Correspondence: Dr. Shorifa Shahjadi, Assistant Professor, Department of Physiology, Bangabandhu Sheikh Mujib Medical University, Dhaka. Cell Phone: 01711560441, e mail: shahjadi79@ gmail.com 
have mitochondrial dysfunction ${ }^{8}$. The number of mitochondria in a cell is directly proportional to the energy requirement of that cell. Skeletal muscle, gastrointestinal system and brain contain large numbers of mitochondria. In addition, mitochondrial dysfunction causes impairment of neuronal function, with imbalance in excitatory and inhibitory neurotransmitters ${ }^{9}$. The mitochondrial disorders are based on clinical, biochemical, molecular and histological findings. Biochemical basis of mitochondrial dysfunction are lactate, ammonia, creatinine kinase (CK), aspartate aminotransferase (AST) and alanine aminotransferase (ALT) level in blood. As in mitochondrial dysfunction TCA cycle impairment occur so conversion of pyruvate to lactate, alanine, TCA cycle intermediates increases and aerobic respiration inhibited. Inhibition of aerobic respiration leads to âoxidation impairment and decreases cellular energy level that lead to increase in anaerobic respiration. So, lactate level increases ${ }^{9}$.

Ammonia is produced in intestine by bacteria during protein metabolism. Liver detoxify ammonia to urea by urea cycle ${ }^{10}$. In this process three ATP molecules needed. In mitochondrial dysfunction ATP level decreases that impairs urea cycle. So ammonia level in blood increases ${ }^{9}$. Increased ammonia level in autism spetrum disorder children may lead to toxicity and ATP depletion in brain ${ }^{11}$.

Mitochondrial dysfunction causes loss of some cellular integrity of certain organ such as muscle and liver. Enzymes of these organs comes out of cell. So serum creatinine kinase (CK), serum aspartate aminotransferase (AST) and alanine aminotransferase (ALT) rises ${ }^{9}$. Some researcher found increased ammomia and lactate level in a study ${ }^{12}$. Some researcher of USA found elevation of serum aspartate aminotransferase (AST) in 38\% of autism spetrum disorder children with mitochondrial dysfunction and in 15\% of normal children. Elevation of serum creatinine kinase (CK) also found in $47 \%$ of these children ${ }^{13}$.

With the best of our knowledge, no published data is available in our country. The outcome of this study will reveal the importance of screening of the mitochondrial dysfunction in autism spectrum disorder children. The result of this study may help the clinician in early diagnosis of mitochondrial dysfunction in autism spetrum disorder children and help in diet planning for these children.

\section{Materials and Methods}

10 diagnosed children of Autism Spectrum disorder (Group B), with age range 3-5 year were taken for this analytical type of case-control study. 10 age and BMI matched apparently healthy children were taken as control (Group A). This study was conducted in the Department of Physiology of Bangabandhu Sheikh Mujib Medical University (BSMMU), Shahbag, Dhaka. The study group was selected from the Parent's Forum (DOHS, Mohakhali) for autism spectrum disorder children and the control group was selected from some normal school. After selection of the subject, thorough information was given to their parents about the objective and study procedure. Their parents were encouraged for voluntary participation of their children. Their parents were also allowed freedom to withdraw their children from the study even after participation whenever they feel like. When their parents were agreed for participation then an informed written consent was obtained from their parents. The parents of subject were requested to attend the Department of Physiology of BSMMU, Dhaka for examination of their children. Detail personal, medical, family, socioeconomic, occupational and dietary histories of the children were recorded in a data schedule from their parents. Thorough physical examinations of the subject were done. Anthropometric measurement including age and BMI were taken. Then $5 \mathrm{ml}$ of venous blood were collected from ante-cubital vein from each subject of both groups for estimation of biochemical variables. Immediately after taking the blood, blood ammonia was measured in the laboratory of the BIRDEM. Then serum lactate, creatine kinase (CK), aspartate aminotransferase (AST) and alanine aminotransferase (ALT) level were measured in the laboratory of the Biochemistry Department, BSMMU. Data were expressed in mean \pm SE and also in percentage. Statistical 
analysis was done by using SPSS for windows version 16. Independent samples ' $t$ ' test were used as the tests of significance. P value $<0.05$ was accepted as significant.

\section{Results}

In this study, the mean value of blood ammonia, serum lactate, serum aspartate aminotransferase and creatine kinase levels were significantly higher in the study group in comparison to that of healthy control children $(p<0.001)$. Percentages of elevated serum enzymes level are summarized in Table-II. In this study, blood ammonia, serum lactate, AST, ALT and CK level were higher in $9(90 \%)$, $8(80 \%), 3(30 \%), 0(0 \%)$ and $4(40 \%)$ subjects of study group. But blood ammonia level were higher in only $1(10 \%)$ and no rise of serum lactate, AST, ALT and CK level in control group respectively.

Table- I

Characteristics of the study subjects.

\begin{tabular}{lccc}
\hline Parameters & $\begin{array}{c}\text { Neurodevelopmental } \\
\text { disorder children }(\mathrm{n}=50)\end{array}$ & $\begin{array}{c}\text { Healthy children } \\
(\mathrm{n}=50)\end{array}$ & $\begin{array}{c}p \\
\text { value }\end{array}$ \\
\hline Blood Ammonia & $50.50 \pm 4.15$ & $22.60 \pm 2.16$ & $0.000^{* * *}$ \\
(ìmol/1) & $(20-71)$ & $(15-38)$ & \\
Serum & $32.35 \pm 7.44$ & $12.50 \pm 0.92$ & $0.026^{*}$ \\
Lactate (mg/dl) & $(16-56)$ & $(8-17)$ & \\
Serum AST (U/L) & $36.60 \pm 3.87$ & $24.80 \pm 1.94$ & $0.017^{*}$ \\
& $(17-63)$ & $(15-35)$ & \\
Serum ALT (U/L) & $34.90 \pm 2.65$ & $33.60 \pm 3.55$ & $0.773^{\mathrm{ns}}$ \\
& $(28-81)$ & $(17-56)$ & \\
Serum CK (U/L) & $187.30 \pm 11.89$ & $134.76 \pm 5.69$ & $0.000^{* * *}$ \\
& $(70-456)$ & $(67-230)$ & \\
\hline
\end{tabular}

Data are expressed as Mean \pm SE. For statistical analysis, independent student' $t$ ' test was performed for comparison between study (autism spectrum disorder children) and control (healthy children) group. Figures in parentheses indicate ranges.

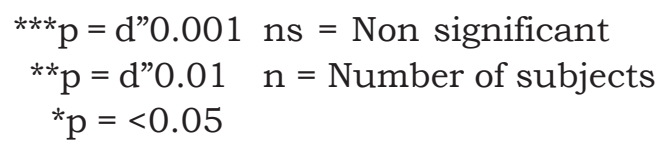

Table- II

Percentage of study and control groups with elevated serum enzymes

\begin{tabular}{lcc}
\hline Parameters & $\begin{array}{c}\text { Neurodevelopmental disorder } \\
\text { children }(\mathrm{n}=50)\end{array}$ & $\begin{array}{c}\text { Healthy } \\
\text { children }(\mathrm{n}=50)\end{array}$ \\
\hline Elevated blood ammonia no. (\%) & $9(90 \%)$ & $1(10 \%)$ \\
Elevated serum lactate no. (\%) & $8(80 \%)$ & $0(0 \%)$ \\
Elevated serum AST no. (\%) & $3(30 \%)$ & $0(0 \%)$ \\
Elevated serum ALT no. (\%) & $0(0 \%)$ & $0(0 \%)$ \\
Elevated serum CK no. (\%) & $4(40 \%)$ & $0(0 \%)$ \\
\hline
\end{tabular}

Figure in parenthesis indicate percentage. ' $n$ ' indicate total number of subjects. 


\section{Discussion}

The present study was undertaken to observe some biochemical variables in children with autism spectrum disorder in order to evaluate their mitochondrial dysfunction. Mitochondrial dysfunction were assessed by measuring blood ammonia, serum lactate, creatine kinase (CK), aspartate aminotransferase (AST) and alanine aminotransferase (ALT) level in autism spectrum disorder children. All these variables were also studied in apparently healthy age and sex matched normal children for comparison. In this study, values of all the biochemical variables of healthy subjects were within physiological limit and were almost similar to those reported by different investigators abroad $13,14,15$.

In this study, blood ammonia level was higher in the study group compared to control group. Almost similar findings were observed by several investigators from different countries $^{12,16}$ In this present study, blood ammonia level was significantly higher in the study group $(\mathrm{p}<0.001)$ than those of control group. In addition, blood ammonia level was found abnormally high in $90 \%$ children of study group and in $10 \%$ children of control group. Some investigators also observed increased blood ammonia level in $78 \%$ of autism spectrum disorder children ${ }^{12}$.

In our study, serum lactate level was higher in the study group compare to control group. Almost similar types of findings were also reported by several studies $9,15,17,18$. In this present study, increased serum lactate level was found in $80 \%$ of study group, whereas serum lactate level was found within normal range in the entire control group. Similarly, increased levels of serum lactate were reported as $30 \%, 35 \%, 76.7 \%$ and $76 \%$ of autism spectrum disorder children ${ }^{9,14,15,19 .}$

In our study, serum aspartate aminotransferase (AST) level was higher in the study group compare to control group and this supported by some studies ${ }^{9}, 13$. In this present study, serum aspartate aminotransferase (AST) level was significantly higher in the study group than those of control group. In addition, increased serum AST level was found in 30\% of study group, whereas serum AST level was found within normal range in the entire control group. Some investigator also found elevated serum AST level in 38\% and 36\% of autistic spectrum disorders children ${ }^{13}, 15$.

In this study, serum alanine aminotransferase (ALT) level was not higher in the study group compared to control group. Several investigators reported elevated serum alanine aminotransferase level in $52 \%$ of autism spectrum disorder children ${ }^{15}$.

In this present study, serum creatine kinase (CK) level was higher in the study group compared to control group. Almost same findings were reported by several investigators from different countries ${ }^{13}$. Moreover, serum creatine kinase (CK) level was found significantly higher in the study group than those of control group. Again, serum CK level was found higher in $40 \%$ of study group, whereas serum CK level was found within normal range in the entire control group. Some reaserchers also found elevated serum CK level in $47 \%$ and $28 \%$ of autism spectrum disorder children ${ }^{13,15}$.

\section{Conclusion}

From the result of this case-control type of study, it may be concluded that mitochondrial dysfunction occur in children with autism spectrum disorder children.

\section{References:}

1. Al-Mosalem O, El-Ansary A, Attas O, Al-Ayadhi L. Metabolic biomarkers related to energy metabolism in Saudi autistic children. Clin Biochem 2009; 42:949-957.

2. Alison C. Herndon, Carolyn DiGuiseppi, Susan L. Johnson, Jenn Leiferman, Ann Reynolds. Does nutritional intake differ between children with autism spectrum disorders and children with typical development? J Autism Dev Disord 2009; 39: 212-222.

3. Reichelt K L, Tveiten D, Knivsberg A, Bronstad G. Peptides role in autism with emphasis on exorphins. Microbial Ecology in Health \& Disease 2012; 23:18958

4. Allsopp MY, Rice C, Karapurkar T, Doernberg N, Boyle C \& Murphy C. Prevalence of autism in a US metropolitan area. The Journal of the American Medical Association 2003; 289 (1): 49-55. 
5. American Psychiatric Association 1994, Diagnostic and statistical manual of mental disorders, $4^{\text {th }}$ edn.,

6. Bloom ED, Lord C, Zwaigenbaum L, Courchesne E, Dager SR, Schmitz C, et al. The developmental neurobiology of autism spectrum disorder. The Journal of Neuroscience 2006 ; 26 (2): 6897-6906.

7. Pastural E, Ritchie S, Lu Y, Jin W, Kavianpour A, Su-Myat K K, et al. Novel plasma phospholipid biomarkers of autism: Mitochondrial dysfunction as a putative causative mechanism, Prostaglandins, Leukotrines and Essential Fatty Acids 2009 ; 81(4) : 253-264.

8. Giulivi C, Zhang YF, Omanska-Klusek A, RossInta $\mathrm{C}$, Wong $\mathrm{S}$, Hertz-Picciotto $\mathrm{I}$, et al. Mitochondrial dysfunction in autism. The Journal of the American Medical Association 2010;304 (21):2389-2396.

9. Rossignol DA, Bradstreet JJ. Evidence of mitochondrial dysfunction in autism and implications for treatment. A $\mathrm{J}$ Biochem Biotechnol 2008; 4(2): 208-217.

10. Milner JA. Metabolic aberrations associated with arginine deficiency. J Nutr 1985; 115: 516-523.

11. Kosenko E, Kaminsky Y, Grau E, et al. Brain ATP depletion induced by acute ammonia intoxication in rats is mediated by activation of the NMDA receptor and $\mathrm{Na}^{+}, \mathrm{K}^{+}$-ATPase. J Neurochem 1994; 63(6): 2172-8.

12. Filipek PA, Juranek J, Nguyen MT, Cummings C, Gargus JJ. Relative Carnitine Deficiency in Autism. Journal of Autism and Developmental Disorders 2004; 34(6): 615-623.
13. Poling JS, Frye RE, Shoffner J, Zimmerman AW. Developmental regression and dysfunction in children mitochondrial with autism. J Child Neurol 2006; 21: 170-172.

14. Mostafa GA, E1-Gamal HA, E1-Wakkad ASE, E1Shorbagy OE, Hamza MM. Polyunsaturated Fatty Acids, Carnitine and Lactate as a Biological markers of Brain Energy in Autistic Children. Int J Ch Neuropsychiatry 2005; 2(2): 179-188.

15. Weissman JR, Kelly RI, Bauman ML, Cohen BH, Murray KF, Mitchell RL, Kern RL, Natowicz MR. Mitochondrial disease in autism spectrum disorder patients: a cohort analysis. PLoS One 2008; 3(11): 3815 .

16. Bradstreet J J, Smith S, Rossignol D A, Baral M. Biomarker-Guided Interventions of Clinically Relevant Conditions Associated With autistic spectrum disorders and Attention Deficit Hyperactivity Disorder. Alternative Medicine Review 2010; 15(1): 15-32.

17. Marin-Garcia J, Ananthakrishnan R, Goldenthal MJ, Filiano JJ, Sarnat HB. Skeletal muscle mitochondrial defects in nonspecific neurologic disorders. Pediatr Neurol 1999; 21(2): 538-42.

18. Correia C, Coutinho AM, Diogo L, et al. Brief report: high frequency of biochemical markers for mitochondrial dysfunction in autism: no association with the mit0ochondrial aspartate/ glutamate carrier SLC25A12 gene. J Autism Dev Disord 2006; 36:1137-1140.

19. Rossignol DA, Frye RE. Mitochondrial dysfunction in autism spectrum disorders: a systemic review and meta-analysis. Mol Psychiatry 2012; 17(3): 290-314. 